\title{
Long-term prognosis of patients with systemic lupus erythematosus-associated pulmonary arterial hypertension: CSTAR-PAH cohort study
}

\author{
Junyan Qian ${ }^{1,18}$, Mengtao $\mathrm{Li}^{1,18}$, Xiao Zhang ${ }^{2,18}$, Qian Wang ${ }^{1}$, Jiuliang Zhao ${ }^{1}$, \\ Zhuang Tian $^{3}$, Wei Wei ${ }^{4}$, Xiaoxia Zuo ${ }^{5}$, Miaojia Zhang ${ }^{6}$, Ping Zhu ${ }^{7}$, Shuang Ye ${ }^{8}$, \\ Wei Zhang ${ }^{9}$, Yi Zheng ${ }^{10}$, Wufang $\mathrm{Qi}^{11}$, Yang $\mathrm{Li}^{12}$, Zhuoli Zhang ${ }^{13}$, Feng Ding ${ }^{14}$, \\ Jieruo $\mathrm{Gu}^{15}$, Yi Liu ${ }^{16}$, Yanhong Wang ${ }^{17}$ and Xiaofeng Zeng ${ }^{1}$
}

@ERSpublications

The long-term prognosis of SLE-associated PAH is newly reported by a large multicentre cohort study http://ow.ly/FRaS30mNAZA

Cite this article as: Qian J, Li M, Zhang X, et al. Long-term prognosis of patients with systemic lupus erythematosus-associated pulmonary arterial hypertension: CSTAR-PAH cohort study. Eur Respir J 2019; 53: 1800081 [https://doi.org/10.1183/13993003.00081-2018].

ABSTRACT This study aimed to identify the long-term clinical outcomes and prognostic factors of patients with systemic lupus erythematosus (SLE)-associated pulmonary arterial hypertension (PAH) confirmed by right heart catheterisation.

A multicentre prospective cohort of SLE-associated PAH was established. Baseline and follow-up records were collected. The primary end-point was death. The secondary exploratory end-point was treatment goal achievement (TGA), defined as an integrated outcome.

In total, 310 patients were enrolled from $14 \mathrm{PAH}$ centres. The 1-, 3- and 5-year survival rates were $92.1 \%, 84.8 \%$ and $72.9 \%$, respectively. The 1 -, 3 - and 5 -year TGA rates were $31.5 \%, 53.6 \%$ and $62.7 \%$, respectively. Baseline serositis, 6-min walking distance $>380 \mathrm{~m}$ and cardiac index $\geqslant 2.5 \mathrm{~L} \cdot \mathrm{min}^{-1} \cdot \mathrm{m}^{-2}$ were identified as independent prognostic factors of TGA. Patients with baseline serositis were more likely to reach TGA after intensive immunosuppressive therapy. TGA was identified as a positive predictor of survival in patients with SLE-associated PAH.

TGA was associated with long-term survival, which supports the treat-to-target strategy in SLEassociated PAH. Baseline heart function predicted both survival and treatment goal achievement in patients with SLE-associated PAH. Patients with serositis at baseline tended to benefit from intensive immunosuppressive therapy and have a better clinical outcome.

Published online Feb 14 2019; republished Feb 282019 to correct an error in the labelling of figure 4c.

This article has supplementary material available from erj.ersjournals.com

Received: Jan 132018 | Accepted after revision: Nov 122018

Copyright OERS 2019 


\section{Introduction}

Connective tissue disease (CTD) is a major cause of pulmonary arterial hypertension (PAH) and causes $25.3 \%$ of PAH and $49.9 \%$ of associated PAH [1]. However, the underlying CTD varies between different populations. Although systemic sclerosis (SSc) is widely recognised as the major underlying cause of CTD-associated PAH in the Western world $[2,3]$, systemic lupus erythematosus (SLE)-associated PAH is most commonly reported in Asian countries $[4,5]$, especially in China, due to its higher prevalence of SLE than that found in other ethnicities [6]. Notably, according to a meta-analysis of studies from USA $[2,7]$, UK [8], Japan [9] and China [4], the 5-year pooled survival rate was $68 \%$ for patients with SLE-associated PAH [10]. Compared with the 5-year survival rate for patients with SLE $(92-94 \%)[11,12]$, the clinical outcome of SLE patients is dramatically worsened after developing PAH [13]. However, only three studies (including 124 patients) meeting the eligible criteria were used to calculate the 5-year pooled survival rate with a huge heterogeneity $\left(I^{2}=52.7 \%\right)$ [10]. Thus, a large, multicentre study is required to determine the long-term prognosis of patients with SLE-associated PAH.

The Chinese SLE Treatment and Research Group (CSTAR) was established in 2009 to collect data from Chinese patients with SLE and was further extended with the formation of the Chinese Rheumatism Data Center, which is directed by the National Health and Family Planning Commission of the People's Republic of China $[14,15]$. Based on the CSTAR registry, we determined the prevalence of PAH among Chinese patients with SLE [16]. Herein, we identified the long-term clinical outcomes, including mortality and treatment goal achievement (TGA) and related prognostic factors of patients with SLE-associated $\mathrm{PAH}$ confirmed by right heart catheterisation (RHC), and demonstrated the association between mortality and TGA.

\section{Methods}

\section{CSTAR-PAH cohort}

The CSTAR registry is a nationwide registry involving 104 rheumatology centres covering 30 provinces in China [15]. In total, 14 referral centres of CTD-associated PAH were qualified as patient enrolment centres (supplementary material). Patients who visited the referral centres from November 2006 to May 2016 and met the following criteria were enrolled in the CSTAR-PAH cohort. The eligible criteria included the diagnosis of SLE confirmed by the 2012 Systemic Lupus International Collaborating Clinics (SLICC) classification criteria [17] and the diagnosis of PAH based on RHC defined as the mean pulmonary arterial pressure $(\mathrm{mPAP}) \geqslant 25 \mathrm{mmHg}$ at rest, pulmonary arterial wedge pressure (PAWP) $\leqslant 15 \mathrm{mmHg}$ and pulmonary vascular resistance (PVR) $>3$ Wood units [18]. Patients with other types of pulmonary hypertension revealed by a pulmonary function test showing total lung capacity $<60 \%$ and ventilation/perfusion scintigraphy or computed tomographic pulmonary angiography showing pulmonary thromboembolism were excluded. Patients with overlapping CTD, such as SSc or other CTDs were also excluded. In addition, application of PAH guidelines [18, 19] and a census-based SLE-associated PAH treatment regimen [20] were required as inclusion criteria. This study was approved by the institutional review board, and we obtained informed consent from each patient.

Affiliations: ${ }^{1}$ Dept of Rheumatology, Peking Union Medical College Hospital, Peking Union Medical College and Chinese Academy of Medical Sciences, Key Laboratory of Rheumatology and Clinical Immunology, Ministry of Education, Beijing, China. ${ }^{2}$ Dept of Rheumatology, Guangdong General Hospital, Guangzhou, China. ${ }^{3}$ Dept of Cardiology, Peking Union Medical College Hospital, Peking Union Medical College and Chinese Academy of Medical Sciences, Beijing, China. ${ }^{4}$ Dept of Rheumatology, Tianjin Medical University General Hospital, Tianjin, China. ${ }^{5}$ Dept of Rheumatology, Xiangya Hospital, Central South University, Changsha, China. ${ }^{6}$ Dept of Rheumatology, The First Affiliated Hospital of Nanjing Medical University, Nanjing, China. ${ }^{7}$ Dept of Clinical Immunology, PLA Specialised Research Institute of Rheumatology and Immunology, Xijing Hospital, Fourth Military Medical University, Xi'an, China. ${ }^{8}$ Dept of Rheumatology, Ren Ji Hospital South Campus, School of Medicine, Shanghai Jiao Tong University, Shanghai, China. ${ }^{9}$ Dept of Rheumatology, Ren Ji Hospital, School of Medicine, Shanghai Jiao Tong University, Shanghai, China. ${ }^{10}$ Dept of Rheumatology, Beijing ChaoYang Hospital, Capital Medical University, Beijing, China. ${ }^{11}$ Dept of Rheumatology, the First Central Hospital, Tianjin, China. ${ }^{12}$ Dept of Rheumatology, the Second Affiliated Hospital of Harbin Medical University, Harbin, China. ${ }^{13}$ Dept of Rheumatology and Clinical Immunology, Peking University First Hospital, Beijing, China.

${ }^{14}$ Dept of Rheumatology, Qilu Hospital of Shandong University, Jinan, China. ${ }^{15}$ Dept of Rheumatology, the Third Affiliated Hospital of Sun Yat-sen University, Guangzhou, China. ${ }^{16}$ Dept of Rheumatology and Immunology, West China Hospital, Sichuan University, Chengdu, China. ${ }^{17}$ Dept of Epidemiology and Biostatistics, Institute of Basic Medical Sciences, China Academy of Medical Sciences and Peking Union Medical College, Beijing, China. ${ }^{18}$ These authors contributed equally.

Correspondence: Xiaofeng Zeng, Dept of Rheumatology, Peking Union Medical College Hospital, No. 1 Shuaifuyuan, Wangfujing Ave, 100730, Beijing, China. E-mail: xiaofeng.zenglacstar.org.cn 


\section{Data collection}

The time of recruitment (baseline) was defined as the time of SLE-associated PAH diagnosis confirmed by RHC. At baseline, we collected demographic information and medical history and performed the physical examination, laboratory evaluations and transthoracic echocardiography (TTE). Data collected at baseline included age; sex; onset of SLE and PAH; organ involvement; SLE disease activity index [21]; World Health Organization functional class (WHO FC); 6-min walking distance (6MWD); levels of serum brain natriuretic peptide (BNP) and N-terminal pro-BNP (NT-proBNP); anti-U1 ribonucleoprotein status; antiphospholipid antibody status; TTE and RHC parameters (which were reviewed by independent cardiologists), including mPAP, PAWP, PVR, cardiac output, cardiac index and right atrial pressure (RAP); and treatment regimen. The duration of SLE was defined as the time span from the diagnosis of SLE to the diagnosis of SLE-associated PAH by RHC (baseline). Serositis was defined as pleuritis/pleural effusion and/or pericarditis/pericardial effusion detected by TTE, chest radiography or chest computerised tomography scan [17].

\section{Follow-up}

A comprehensive follow-up evaluation of every patient was recorded and reported at least once a year. These measures included WHO FC, 6MWD, serum BNP, serum NT-proBNP and TTE. In addition, treatment regimens were recorded. Death, cause of death, hospitalisation due to disease deterioration, disease-related transplantation or atrial septostomy were also recorded and reported to the database.

\section{Outcomes}

The primary end-point was death from any cause. Mortality was ascertained from PAH centre records and the national population tracking system. The exploratory experimental end-point was achieving the integrated treatment goal recommended by the European Society of Cardiology (ESC) and the European Respiratory Society (ERS) [19]. Treatment goal was defined as achieved when all of the following four aspects were reached. 1) Clinical symptoms: no signs of right heart failure, syncope or progression; 2) WHO FC I or II or 6MWD $>380-440 \mathrm{~m}$; 3) serology: $\mathrm{BNP}<50 \mathrm{ng} \cdot \mathrm{L}^{-1}$ or NT-proBNP $<300 \mathrm{ng} \cdot \mathrm{L}^{-1}$; and 4) cardiac imaging: normal right atrial area according to echocardiography. For socioeconomic reasons, haemodynamic parameters were not available in every follow-up visit and therefore were not included in evaluating TGA in our study. TGA was ascertained according to the follow-up date reported by each PAH centre prior to the course of data analysis.

\section{Statistical analyses}

Quantitative data are described as the mean \pm SD. Nonquantitative data are described as counts and percentages. Cumulative probabilities of survival and not achieving the treatment goal were calculated using the Kaplan-Meier estimator. Further comparisons were performed using a log-rank test. The Kaplan-Meier survival curves comparing patients with TGA and without TGA were plotted by the method proposed by Simon and Makuch [22] for time-dependent covariates. All-cause mortality was used in survival statistics. Follow-up time was calculated from the date of initial diagnostic RHC. Baseline characteristics between patients with and without clinical outcomes (death and TGA) were compared. Baseline factors with clinical significance, including age at recruitment, sex, clinical features, RHC parameters, TTE parameters and treatment selection were analysed by univariate Cox regressions. Cut-offs for cardiac index and 6MWD were based on previous studies and guidelines $[18,19]$. Considering that there is no widely recognised cut-off for PVR, we performed an outcome-oriented statistical analysis for cut-point determination using the method proposed by ConTAL and O'Quigley [23], and used 12 Wood units as the cut-off in our study. Other parameters, including NT-proBNP, BNP and RAP were firstly dichotomised according to tertiles, and then used in the Cox regression. As TGA was a time-dependent covariate, we used time-dependent Cox regression, in order to avoid immortal time bias. Variables with clinical relevance selected by univariate analysis, including age, sex, and others, were further subjected to multivariate analysis. The proportional hazard assumption based on the Schoenfeld residuals was tested. p-values $<0.05$ were considered significant. Data analyses were conducted using SPSS 19.0 (SPSS, Chicago, IL, USA) and R software (www.r-project.org).

\section{Results}

Baseline characteristics of patients with SLE-associated PAH

310 patients with RHC-confirmed SLE-associated PAH were enrolled in our study (table 1). The mean duration of SLE was $4.4 \pm 5.4$ years. $51.7 \%$ patients were WHO FC I or II, and the mean mPAP was 46.5 $\pm 12.1 \mathrm{mmHg}$. $99.4 \%$ and $92.6 \%$ patients were treated with glucocorticoid and immunosuppressant therapy, respectively. $69.4 \%$ of patients were receiving PAH medication: $56.7 \%$ received endothelin receptor antagonists, $58.1 \%$ received phosphodiesterase inhibitors and $7.0 \%$ received prostacyclin analogues. Among the 310 recruited patients, 282 patients with confirmed mortality statuses were included in the survival 
TABLE 1 Baseline characteristics of patients with systemic lupus erythematosus (SLE)-associated pulmonary arterial hypertension (PAH)

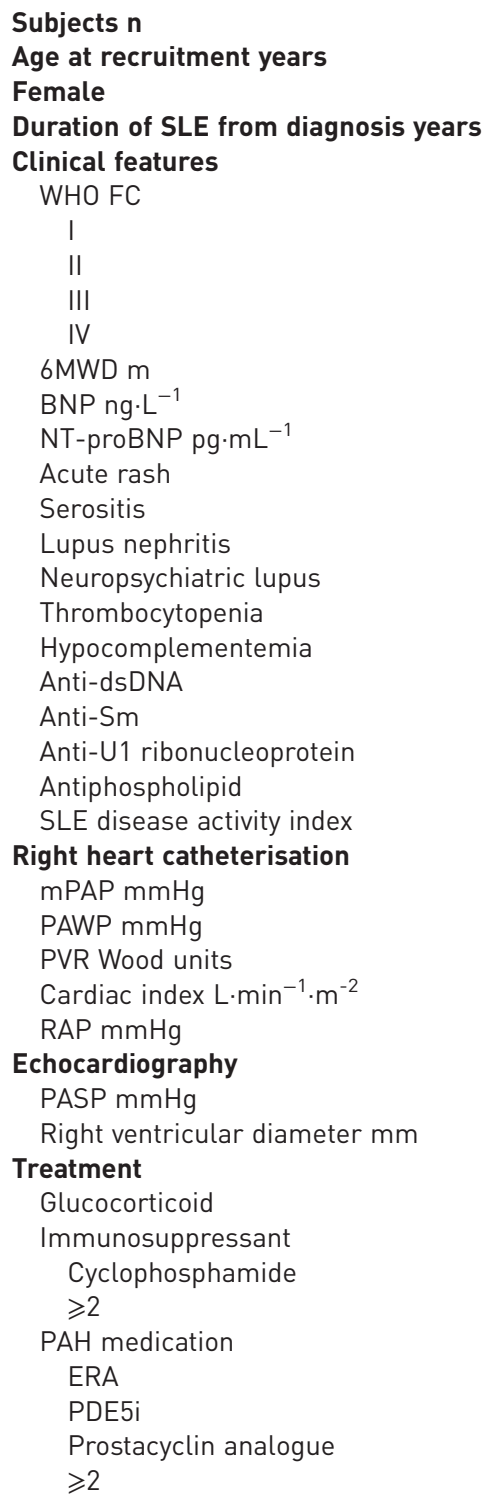

Data are presented as mean \pm SD or \%, unless otherwise stated. WHO FC: World Health Organization functional class; 6MWD: 6-min walking distance; BNP: brain natriuretic peptide; NT-proBNP: N-terminal pro-BNP; mPAP: mean pulmonary arterial pressure; PAWP: pulmonary arterial wedge pressure; PVR: pulmonary vascular resistance; RAP: right atrial pressure; PASP: pulmonary arterial systolic pressure; ERA: endothelin receptor antagonist; PDE5i: phosphodiesterase inhibitor.

analysis, and 263 patients with full follow-up data were included in the TGA study (figure 1). The maximum follow-up duration was 107.5 months, and the median follow-up was 24.0 months.

Survival and predictors of mortality for patients with SLE-associated PAH

During follow-up, 42 (14.9\%) deaths occurred. No PAH-related transplantations or atrial septostomies occurred. The 1-, 3- and 5-year survival rates for patients with SLE-associated PAH were 92.1\%, 84.8\% and $72.9 \%$, respectively (figure $2 \mathrm{a}$ ). The mean survival time was 7.0 years.

Baseline characteristics between survivors and nonsurvivors were compared (supplementary table S1). According to the univariate Cox analysis, the following baseline factors were associated with mortality 


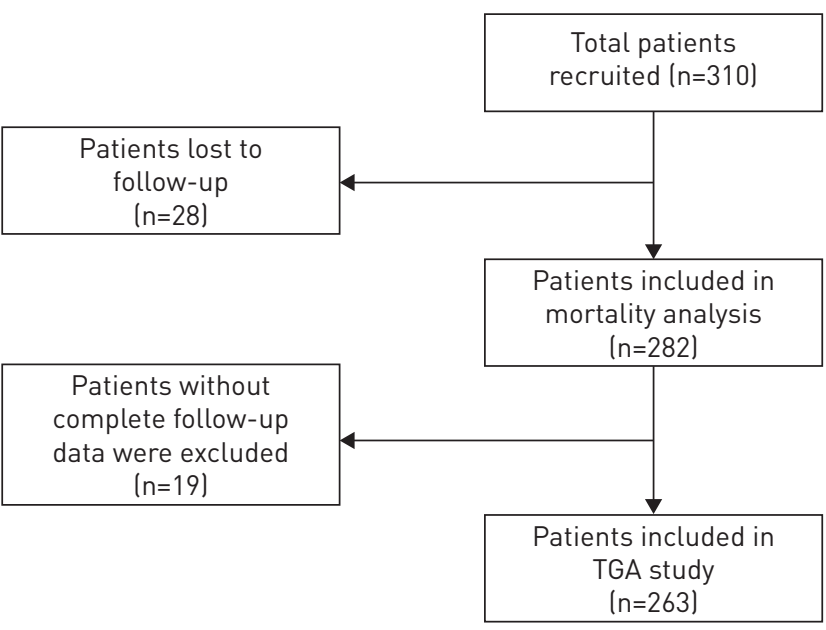

FIGURE 1 Study flow diagram. TGA:

treatment goal achievement.

(table 2). 6MWD $\leqslant 380 \mathrm{~m}$; WHO FC III-IV; NT-proBNP >1695.0 pg.mL $\mathrm{mL}^{-1}$; high mPAP; cardiac index $<2.5 \mathrm{~L} \cdot \mathrm{min}^{-1} \cdot \mathrm{m}^{-2}$ (figure $2 \mathrm{~b}$ ); PVR $>12$ Wood units; and enlarged right ventricular diameter. According to the multivariate Cox regression analysis adjusting sex and age, cardiac index $<2.5 \mathrm{~L} \cdot \mathrm{min}^{-1} \cdot \mathrm{m}^{-2}$ (hazard ratio (HR) 2.62, 95\% CI 1.33-5.18; $\mathrm{p}=0.006$ ) was identified as an independent predictor of mortality for patients with SLE-associated PAH.

\section{Relationship between TGA and death}

TGA was identified as a factor positively associated with survival in univariate time-dependent Cox regression (HR 0.18, 95\% CI 0.05-0.59; $\mathrm{p}=0.005$ ). We then compared the cumulative survival rates between patients who achieved the treatment goal and patients who did not (figure 3). The cumulative survival rate was significantly higher for patients who achieved TGA $(\mathrm{p}=0.002)$.

\section{TGA and prognostic factors of TGA for patients with SLE-associated PAH}

As the secondary exploratory end-point of our study, we identified the TGA status of individual patients according to the follow-up data. Among them, 115 (43.7\%) patients achieved the treatment goal, and their 1-, 3- and 5-year TGA rates were $31.5 \%, 53.6 \%$ and $62.7 \%$, respectively (figure $4 \mathrm{a}$ ). The median time from baseline to TGA was 2.5 years. Baseline characteristics were compared between patients who reached the treatment goals and patients who did not (supplementary table S2). To investigate the predictors of TGA, univariate Cox regression was conducted. Characteristics at baseline, including serositis (figure 4b), $6 \mathrm{MWD}>380 \mathrm{~m}$ (figure 4c), low $\mathrm{mPAP}$, BNP $>339.3 \mathrm{ng} \cdot \mathrm{L}^{-1}$, cardiac index $\geqslant 2.5 \mathrm{~L} \cdot \mathrm{min}^{-1} \cdot \mathrm{m}^{-2}$, PVR $\leqslant 12$ Wood units and a smaller right ventricular diameter were associated with TGA (table 3). After adjusting for the effects of multiple covariates, baseline serositis (HR 1.99, 95\% CI 1.27-3.11; p=0.003), $6 \mathrm{MWD}>380 \mathrm{~m}$ (HR 2.12, 95\% CI 1.22-3.68; $\mathrm{p}=0.008)$ and cardiac index $\geqslant 2.5 \mathrm{~L} \cdot \mathrm{min}^{-1} \cdot \mathrm{m}^{-2}$ (HR 2.14 , 95\% CI 1.26-3.62; $\mathrm{p}=0.005$ ) were identified as independent prognostic factors of TGA (table 3 ).

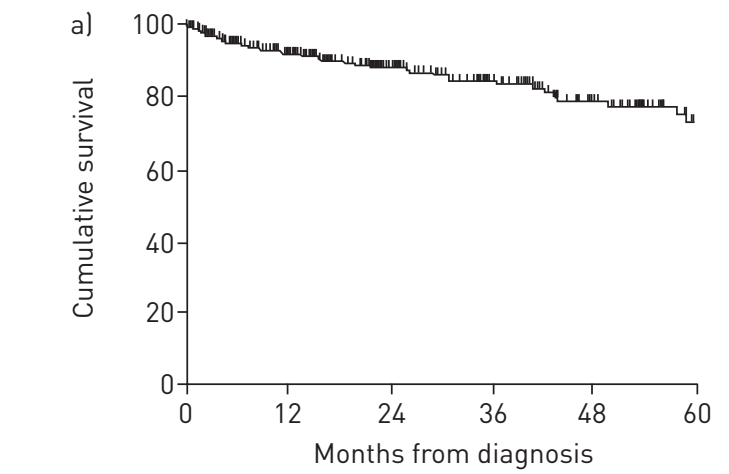

At risk n $\quad 282 \quad 200 \quad 142 \quad 89 \quad 57 \quad 31$

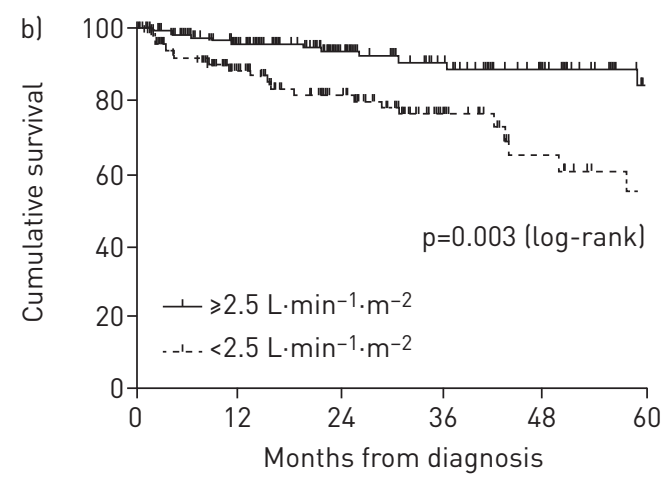

At risk $\mathrm{n}$

Cardiac index $\geqslant 2.5 \mathrm{~L} \cdot \mathrm{min}^{-1} \cdot \mathrm{m}^{-2} \quad 140 \quad 107 \quad 74 \quad 51 \quad 35 \quad 18$

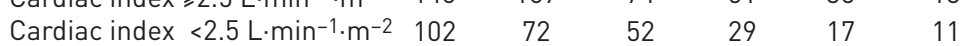

FIGURE 2 Cumulative survival rates for patients with systemic lupus erythematosus (SLE)-associated pulmonary arterial hypertension (PAH). a) All patients with SLE-associated $\mathrm{PAH}$; b) comparison between patients with cardiac index $\geqslant 2.5 \mathrm{~L} \cdot \mathrm{min}^{-1} \cdot \mathrm{m}^{-2}$ and $\mathrm{cardiac}$ index $<2.5 \mathrm{~L} \cdot \mathrm{min}^{-1} \cdot \mathrm{m}^{-2}$. 
Patients with serositis at baseline had better TGA after intensive immunosuppressive therapy We hypothesised that patients with serositis at baseline would respond better to intensive immunosuppressive therapy (IST), defined as therapy with one or more immunosuppressants, including cyclophosphamide, mycophenolate mofetil, cyclosporine A and tacrolimus, than those without serositis at baseline. There were no significant differences in baseline characteristics between patients on IST and those on only

TABLE 2 Predictors of mortality for patients with systemic lupus erythematosus (SLE)-associated pulmonary arterial hypertension

\begin{tabular}{|c|c|c|c|c|}
\hline & \multicolumn{2}{|c|}{ Univariate analysis } & \multicolumn{2}{|c|}{ Multivariate analysis } \\
\hline & $\operatorname{HR}(95 \% \mathrm{CI})$ & $p$-value & $\operatorname{HR}(95 \% \mathrm{CI})$ & p-value \\
\hline $6 M W D \leqslant 380 \mathrm{~m}$ & $4.56(2.05-10.18)$ & $<0.001 * *$ & & \\
\hline WHO FC III-IV & $2.51(1.24-5.09)$ & $0.010^{* *}$ & & \\
\hline \multicolumn{5}{|l|}{ NT-proBNP\# } \\
\hline$<536.3 \mathrm{pg} \cdot \mathrm{mL}^{-1}$ & Reference & & & \\
\hline $536.3-1695.0 \mathrm{pg} \cdot \mathrm{mL}^{-1}$ & $1.26(0.38-4.15)$ & 0.704 & & \\
\hline$>1695.0 \mathrm{pg} \cdot \mathrm{mL}^{-1}$ & $4.78(1.73-13.25)$ & $0.003^{* *}$ & & \\
\hline mPAP $\mathrm{mmHg}$ & $1.04(1.02-1.06)$ & $0.001 * *$ & & \\
\hline Cardiac index $<2.5 \mathrm{~L} \cdot \mathrm{min}^{-1} \cdot \mathrm{m}^{-2}$ & $2.82(1.43-5.55)$ & $0.003^{* *}$ & $2.62(1.33-5.18)$ & $0.006 *$ \\
\hline \multirow{2}{*}{\multicolumn{5}{|c|}{ RAP $^{\#}$}} \\
\hline & & & & \\
\hline$<3 \mathrm{mmHg}$ & Reference & & & \\
\hline $3-7 \mathrm{mmHg}$ & $0.78(0.29-2.04)$ & 0.61 & & \\
\hline$>7 \mathrm{mmHg}$ & $1.29(0.52-3.18)$ & 0.58 & & \\
\hline Missing & $1.36(0.62-3.00)$ & 0.45 & & \\
\hline Right ventricular diameter $\mathrm{mm}$ & $1.04(1.01-1.07)$ & $0.019 *$ & & \\
\hline Anti-U1 ribonucleoprotein positivity & $1.46(0.76-2.81)$ & 0.260 & & \\
\hline Antiphospholipid antibody positivity & $0.69(0.27-1.79)$ & 0.449 & & \\
\hline SLE disease activity index & $0.97(0.91-1.03)$ & 0.251 & & \\
\hline
\end{tabular}

HR: hazard ratio; 6MWD: 6-min walking distance; WHO FC: World Health Organization functional class; NT-proBNP: N-terminal pro-brain natriuretic peptide; mPAP: mean pulmonary arterial pressure; PVR: pulmonary vascular resistance; RAP: right atrial pressure. " : variables grouped into tertiles, where appropriate; ๆ : $30 \%$ of patients had missing data. *: $p \leqslant 0.05 ;{ }^{* *}: p \leqslant 0.01$.

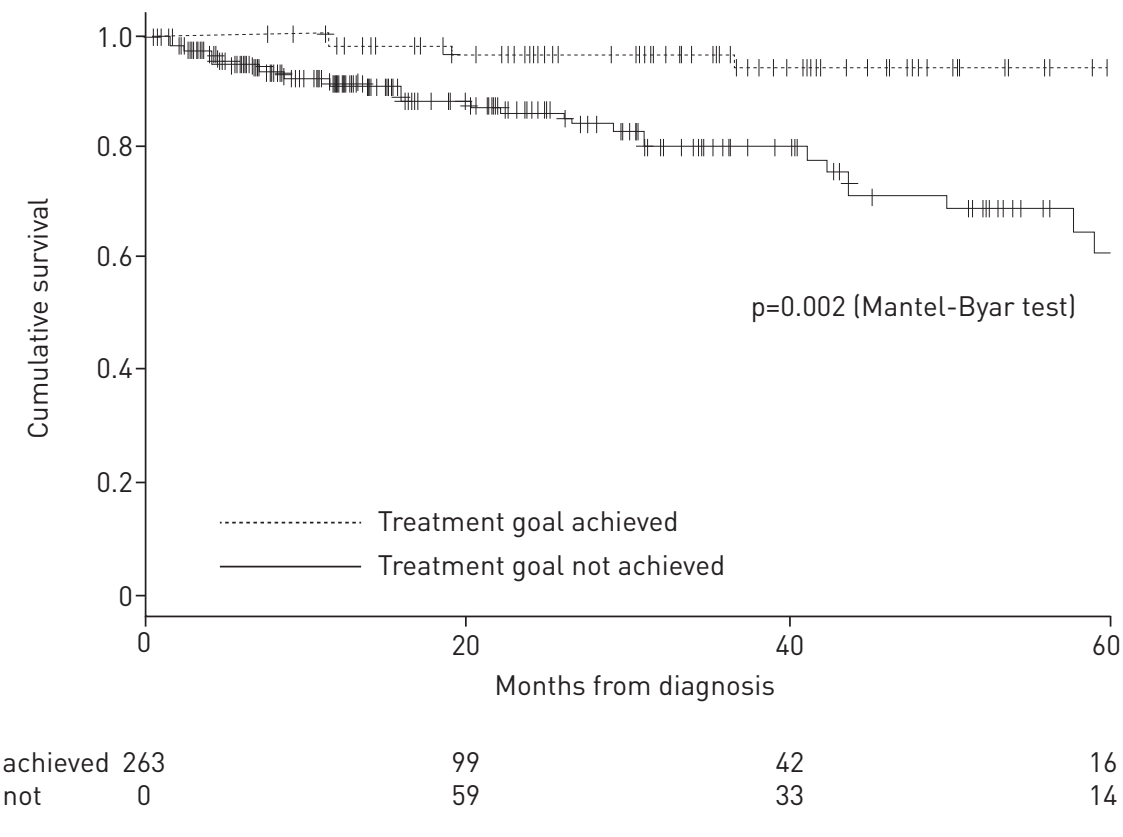

At risk $\mathrm{n}$

Treatment goal achieved 263

Treatment goal not

achieved

FIGURE 3 Comparison of the cumulative survival rates in patients who did and who did not achieve treatment goal. 

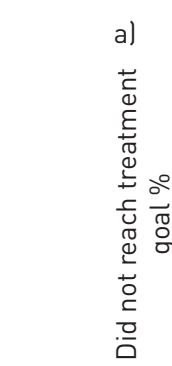

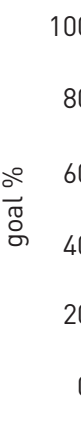
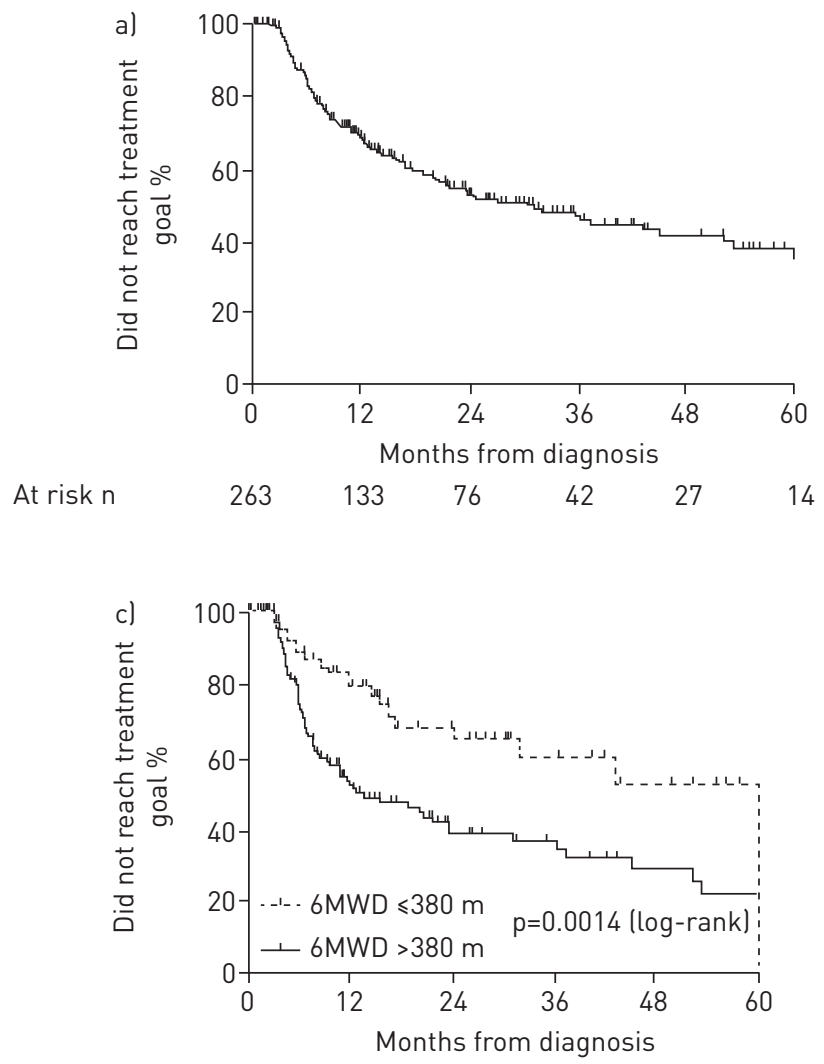

At risk $n$

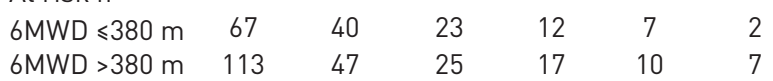

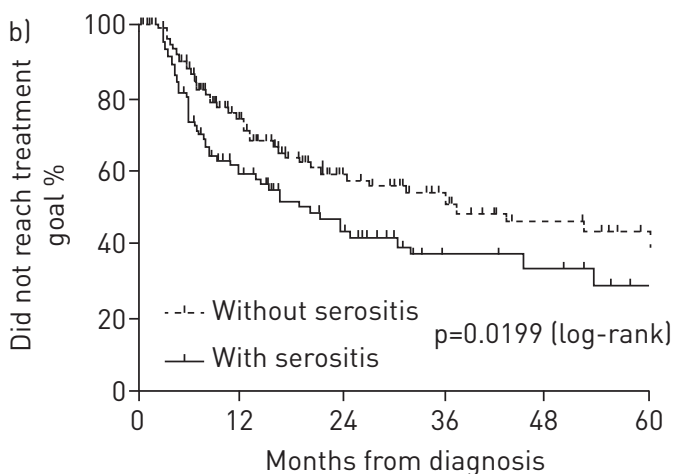

At risk $n$

$\begin{array}{lllllll}\text { Without serositis } & 164 & 86 & 49 & 30 & 18 & 10\end{array}$ With serositis $\quad 99 \quad 49 \quad 27 \quad 13 \quad 10 \quad 5$

FIGURE 4 Cumulative rates of non-achievement of treatment goals (TGA) for patients with systemic lupus erythematosus (SLE)-associated pulmonary arterial hypertension (PAH). a) Cumulative non-TGA rates for all patients with SLE-associated PAH; b) comparison between patients with and without baseline serositis; c) comparison between patients with 6MWD $\leqslant 380 \mathrm{~m}$ and $6 \mathrm{MWD}>380 \mathrm{~m}$.

hydroxychloroquine therapy when stratified by baseline serositis (supplementary table S3). Notably, when comparing TGA between the two therapeutic groups, patients with baseline serositis were more likely to reach TGA after IST compared to non-IST recipients (HR 2.47, 95\% CI 1.21-4.17; p=0.013), which was not seen in those without baseline serositis (figure 5).

\section{Discussion}

We identified the prognostic factors for patients with SLE-associated PAH based on a multicentre cohort with RHC-based diagnoses. All-cause mortality and TGA were set as the long-term and medium-term clinical end-points, respectively. The 1-, 3- and 5-year survival rates of patients with SLE-associated PAH were $92.1 \%, 84.8 \%$ and $72.9 \%$, respectively. The $1-, 3-$, and 5 -year survival rates of 1494 SLE patients recruited during the same time period were $98.3 \%, 96.9 \%$ and $95.7 \%$ respectively in China (unpublished data). The survival rates of SLE-associated PAH are higher than those calculated in the previous meta-analysis (1-year 88\%, 3-year 81\%, 5-year 68\%) [10]. In a recent French cohort, the 1-, 3- and 5-year survival rates were $94.1 \%, 89.4 \%$ and $83.9 \%$, respectively [24]. There may be a population difference regarding prognosis; however, time-lead bias, disease severity, therapeutic strategy and the study design should also be considered. According to univariate and multivariate analyses, baseline cardiac index $<2.5 \mathrm{~L} \cdot \mathrm{min}^{-1} \cdot \mathrm{m}^{-2}$ was an independent predictor of mortality. Our study, consistent with the conclusions of studies on idiopathic PAH and SSc-associated $\mathrm{PAH}$, confirmed that heart function, represented by cardiac index, is critical to the clinical outcomes of patients with SLE-associated PAH [25-27]. Preservation or improvement of heart function may become the priority of clinicians in the management of SLE-associated PAH, which will need to be proven in future studies.

Treat-to-target, defined as a therapeutic strategy aiming to treat patients to a goal and improve disease outcome, is increasingly accepted in disease management. A treat-to-target strategy for SLE has been recommended by an international task force, which asserted that complete remission is the main target of 
TABLE 3 Predictors of treatment goal achievement for patients with systemic lupus erythematosus-associated pulmonary arterial hypertension

\begin{tabular}{|c|c|c|c|c|}
\hline & \multicolumn{2}{|c|}{ Univariate analysis } & \multicolumn{2}{|c|}{ Multivariate regression } \\
\hline & HR $(95 \% \mathrm{CI})$ & p-value & $\operatorname{HR}(95 \% \mathrm{CI})$ & p-value \\
\hline Serositis & $1.55(1.07-2.23)$ & $0.021 *$ & $1.99(1.27-3.11)$ & $0.003 * *$ \\
\hline $6 M W D>380 \mathrm{~m}$ & $1.49(1.16-1.92)$ & $0.002^{* *}$ & $2.12(1.22-3.68)$ & $0.008^{* *}$ \\
\hline \multicolumn{5}{|l|}{ BNP $^{\#}$} \\
\hline$<105.3 \mathrm{ng} \cdot \mathrm{L}^{-1}$ & Reference & & & \\
\hline $105.3-339.3 \mathrm{ng} \cdot \mathrm{L}^{-1}$ & $0.83(0.48-1.43)$ & 0.50 & & \\
\hline$>339.3 \mathrm{ng} \cdot \mathrm{L}^{-1}$ & $0.29(0.15-0.57)$ & $<0.001^{* *}$ & & \\
\hline mPAP $\mathrm{mmHg}$ & $0.97(0.96-0.99)$ & $<0.001^{* *}$ & & \\
\hline Cardiac index $\geqslant 2.5 \mathrm{~L} \cdot \mathrm{min}^{-1} \cdot \mathrm{m}^{-2}$ & $1.97(1.29-3.01)$ & $0.002^{* *}$ & $2.14(1.26-3.62)$ & $0.005^{* *}$ \\
\hline PVR $\leqslant 12$ WU & $1.98(1.27-3.10)$ & $0.003^{* *}$ & & \\
\hline \multicolumn{5}{|l|}{ RAP $^{\#}$} \\
\hline$<3 \mathrm{mmHg}$ & Reference & & & \\
\hline $3-7 \mathrm{mmHg}$ & $0.76(0.48-1.22)$ & 0.25 & & \\
\hline$>7 \mathrm{mmHg}$ & $0.45(0.26-0.78)$ & $0.004^{* *}$ & & \\
\hline Missing & $0.29(0.17-0.50)$ & $<0.001^{* *}$ & & \\
\hline
\end{tabular}

HR: hazard ratio; 6MWD: 6-min walking distance; BNP: brain natriuretic peptide; mPAP: mean pulmonary arterial pressure; PVR: pulmonary vascular resistance; RAP: right atrial pressure. \#: variables grouped into tertiles, where appropriate; ${ }^{~}: 30 \%$ of patients had missing data. ${ }^{*}: p \leqslant 0.05 ;{ }^{* *}: p \leqslant 0.01$.

SLE [28]. With regard to the heterogeneity of SLE, the treatment goal may vary in situations with different organ involvement [29]. An integrated criterion with multiple parameters, including WHO FC, symptom, serological, echocardiographic and RHC parameters, was recommended as treatment goals for patients with PAH by ESC/ERS [19]. Although the goal-orientated treatment approach has not been fully validated, several studies have shown recently that PAH patients in a "low-risk" group showed a decreased mortality risk [30-32]. Although RHC is a relatively direct and accurate way of evaluating PAH, RHC cannot be performed in China at every follow-up visit due to its invasive nature and socioeconomic factors. Thus, TGA without RHC parameters was defined as the exploratory secondary end-point in our study, and it was favourably associated with survival among patients with SLE-associated PAH. Our study demonstrated that TGA was associated with the long-term survival and that TGA could be a target in the management of SLE-associated PAH. The long-term survival of patients meeting different numbers of treatment goal at specific time points need to be further compared. The 1-, 3- and 5-year TGA rates were 31.5\%, 53.6\% and $62.7 \%$, respectively. Baseline $6 \mathrm{MWD} \geqslant 380 \mathrm{~m}$ and cardiac index $\geqslant 2.5 \mathrm{~L} \cdot \mathrm{min}^{-1} \cdot \mathrm{m}^{-2}$ were identified as

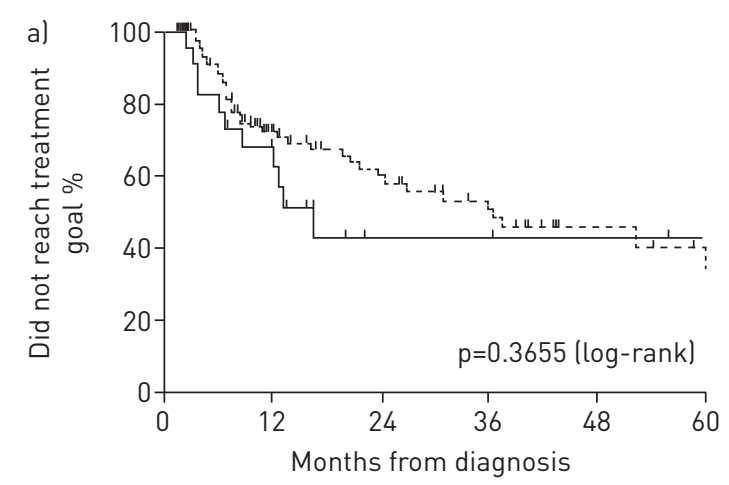

At risk $n$ IST HCQ only

$\begin{array}{llcccc}93 & 47 & 31 & 21 & 11 & 7 \\ 24 & 13 & 4 & 4 & 3 & 2\end{array}$

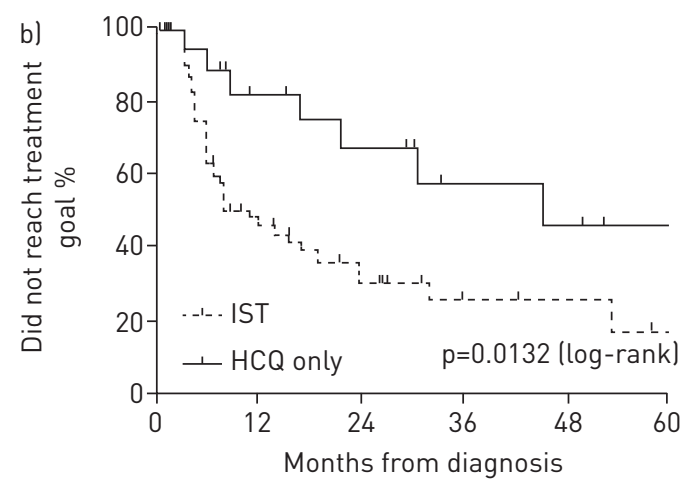

At risk $n$

$\begin{array}{lllllll}\text { IST } & 59 & 23 & 12 & 5 & 4 & 2 \\ \text { HCQ only } & 19 & 13 & 10 & 6 & 5 & 3\end{array}$

FIGURE 5 Cumulative rates of not achieving treatment goals for patients with systemic lupus erythematosus-associated pulmonary arterial hypertension. a) Comparison between patients with intensive immunosuppressive therapy (IST) and patients receiving hydroxychloroquine (HCQ) only among those without baseline serositis; b) comparison between patients receiving IST and patients receiving HCQ only among those with baseline serositis. 
predictors of TGA among patients with SLE-associated PAH in our study, indicating that heart function is crucial in the response to treatment and to even achieving the treatment goal.

Notably, baseline serositis was newly identified to predict TGA in our study. It has been reported in our previous study that SLE disease activity scores were significantly higher in patients with serositis, and PAH was significantly associated with serositis in patients with SLE [33]. Here, we found that indicators of heart failure, such as WHO FC, cardiac index and RAP, had no significant differences in SLE-associated PAH patients with versus without serositis (data not shown), indicating that inflammation may be the major mechanism leading to serositis in patients with SLE-associated PAH in our study. This result raised the question of whether patients with high SLE disease activity, indicated by baseline serositis, could benefit from immunosuppressive therapy and achieve better clinical outcomes. Therefore, a further stratification analysis was conducted. It showed that patients with baseline serositis were more likely to reach TGA after IST than those without IST, while the same phenomenon was not shown in patients without baseline serositis. This result suggests that, in developing future treatment strategies, that baseline serositis may be treated as an indicator for clinicians to initiate intensive IST for patients with SLE-associated PAH. Although the literature is limited, this result concurs with a recent case report finding that a patient with SLE-associated PAH and high disease activity has totally recovered after methylprednisolone pulse therapy [34]. In another recent study, the use of immunosuppressants was an independent predictor of a short-term response in patients with CTD-associated PAH [35]. The potential clinical significance still needs further investigation and evidence from randomised clinical trials.

This study has several limitations. First, 310 patients were initially enrolled in our study, and patients without information about death or TGA were excluded from the prognosis analyses. Fortunately, there was no significant difference in the baseline characteristics of patients included and excluded in the prognosis analyses (data not shown), indicating the lack of the outcome data was random. Second, we included both the incident and prevalent populations. Patients with incident PAH have poorer survival rates than patients with prevalent disease, according to both the French registry and the Registry to Evaluate Early and Long-term Pulmonary Arterial Hypertension Disease Management (REVEAL) studies $[7,36]$. Thus, the incident cohort and prevalent cohort need to be studied separately in future studies. In addition, we reported that $69.4 \%$ of patients in our cohort received PAH target medications at baseline, explained by socioeconomic reasons. Thus, the effect of different treatment regimens needs to be further studied. Lastly, 31.6\% of RAP values were missing in our cohort, as RAP was not a routinely reported value in some PAH centres. However, it showed a tendency of predicting death even without statistical significance. Future studies need to collect complete information on this value and prove its prognostic significance.

This study is the largest prognostic cohort consisting of patients with SLE-associated PAH based on a RHC algorithm. It reported the 1-, 3- and 5-year survival rates of patients with SLE-associated PAH and further identified cardiac index as a predictor of mortality. We reported for the first time the 1-, 3- and 5 -year rates of achieving treatment goals. Cardiac index, 6MWD and serositis were prognostic factors of TGA. TGA was associated with long-term survival, the first evidence supporting the treat-to-target strategy in SLE-associated PAH. Baseline heart function predicted both survival and TGA in patients with SLE-associated PAH. Patients with serositis at baseline tended to benefit from intensive immunosuppressive therapy and to have better clinical outcomes. Further investigation on the therapeutic strategy specific for SLE-associated PAH is urgently needed.

Acknowledgements: We appreciate Yang Ding (Center for Bioinformatics, School of Life Sciences, Peking University, Beijing, China), for his kind technical support during data analysis. We also appreciate the comments and editing of our manuscript by Andrea Ballmer and her medical team from Actelion (Allschwill, Switzerland).

Author contributions: Conception and design: J. Qian, M. Li, X. Zhang, Q. Wang, J. Zhao, Z. Tian, W. Wei, X. Zuo, M. Zhang, P. Zhu, S. Ye, W. Zhang, Y. Zheng, W. Qi, Y. Li, Z. Zhang, F. Ding, J. Gu, Y. Liu, X. Zeng. Analysis and interpretation: J. Qian, M. Li, X. Zhang, Q. Wang, J. Zhao, Y. Wang. Drafting the manuscript for important intellectual content: J. Qian, M. Li, X. Zhang, X. Zeng.

The following investigators were collaborators in the CSTAR-PAH study: C. Huang, X. Yang, D. Xu (rheumatology), Y. Liu, X. Guo, H. Wang, J. Lai (cardiology), Peking Union Medical College Hospital, Peking Union Medical College and Chinese Academy of Medical Sciences; N. Zhang, K. Yang, Y. Liu (rheumatology), S. Dong (cardiology), Tianjin Medical University General Hospital; Y. Lei, Y. Feng (rheumatology), Guangdong General Hospital; Y. Li, Y. Zhou (rheumatology), Xiangya Hospital, Central South University; Q. Wang (rheumatology), The First Affiliated Hospital of Nanjing Medical University; J. Jia, Q. Han (rheumatology), Xijing Hospital, Fourth Military Medical University; L. Guo, J. Chen (rheumatology), Ren Ji Hospital, Shanghai Jiao Tong University; Y. Zhang, Y. Liu (rheumatology), Beijing Chao-Yang Hospital, Capital Medical University; H. Xu (rheumatology), The First Central Hospital, Tianjin; Y. Sun (rheumatology): The Second Affiliated Hospital of Harbin Medical University; Y. Hao, Y. Fan (rheumatology), Peking University First Hospital; Q. Shu, Y. Wang (rheumatology), Qilu Hospital of Shandong University; Z. Lin 
(rheumatology), The Third Affiliated Hospital of Sun Yat-sen University, Guangzhou; P. Qing (rheumatology), West China Hospital, Sichuan University.

Conflict of interest: None declared.

Support statement: This work was supported by the Chinese National Key Research R\&D Program (grant numbers 2017YFC0907601, 2017YFC0907602, 2017YFC0907603, 2008BAI59B02) and the Chinese National High Technology Research and Development Program, Ministry of Science and Technology (grant number 2012AA02A513). Funding information for this article has been deposited with the Crossref Funder Registry.

\section{References}

1 Badesch DB, Raskob GE, Elliott CG, et al. Pulmonary arterial hypertension: baseline characteristics from the REVEAL Registry. Chest 2010; 137: 376-387.

2 Chung L, Liu J, Parsons L, et al. Characterization of connective tissue disease-associated pulmonary arterial hypertension from REVEAL: identifying systemic sclerosis as a unique phenotype. Chest 2010; 138: 1383-1394.

3 Condliffe R, Kiely DG, Peacock AJ, et al. Connective tissue disease-associated pulmonary arterial hypertension in the modern treatment era. Am J Respir Crit Care Med 2009; 179: 151-157.

4 Hao YJ, Jiang X, Zhou W, et al. Connective tissue disease-associated pulmonary arterial hypertension in Chinese patients. Eur Respir J 2014; 44: 963-972.

5 Jeon $\mathrm{CH}$, Chai JY, Seo YI, et al. Pulmonary hypertension associated with rheumatic diseases: baseline characteristics from the Korean registry. Int J Rheum Dis 2012; 15: e80-e89.

6 D'Cruz DP, Khamashta MA, Hughes GR. Systemic lupus erythematosus. Lancet 2007; 369: 587-596.

7 Chung L, Parsons L, Hassoun PM, et al. Outcomes in a newly diagnosed population of connective tissue disease-associated pulmonary arterial hypertension patients from the REVEAL Registry. Arthritis Rheum 2010; 62: Suppl., 1223.

8 Schreiber BE, Valerio C, Handler C, et al. Diagnosis and prognosis of pulmonary arterial hypertension in SLE and SSC. Rheumatology 2011; 50: i40.

9 Shirai Y, Yasuoka H, Okano Y, et al. Clinical characteristics and survival of Japanese patients with connective tissue disease and pulmonary arterial hypertension: a single-centre cohort. Rheumatology 2012; 51: 1846-1854.

10 Qian J, Wang Y, Huang C, et al. Survival and prognostic factors of systemic lupus erythematosus-associated pulmonary arterial hypertension: a PRISMA-compliant systematic review and meta-analysis. Autoimmun Rev 2016; 15: 250-257.

11 Wang Z, Wang Y, Zhu R, et al. Long-term survival and death causes of systemic lupus erythematosus in China: a systemic review of observational studies. Medicine 2015; 94: e794.

12 Mak A, Cheung MW, Chiew HJ, et al. Global trend of survival and damage of systemic lupus erythematosus: meta-analysis and meta-regression of observational studies from the 1950s to 2000s. Semin Arthritis Rheum 2012; 41: 830-839.

13 Boumpas DT, Austin HR, Fessler BJ, et al. Systemic lupus erythematosus: emerging concepts. Part 1: renal, neuropsychiatric, cardiovascular, pulmonary, and hematologic disease. Ann Intern Med 1995; 122: 940-950.

14 Li M, Tian X, Zhang W, et al. CRDC: a Chinese rheumatology research platform. Clin Rheumatol 2015; 34: $1347-1352$.

15 Li M, Zhang W, Leng X, et al. Chinese SLE Treatment and Research group (CSTAR) registry: I. Major clinical characteristics of Chinese patients with systemic lupus erythematosus. Lupus 2013; 22: 1192-1199.

16 Li M, Wang Q, Zhao J, et al. Chinese SLE Treatment and Research group (CSTAR) registry: II. Prevalence and risk factors of pulmonary arterial hypertension in Chinese patients with systemic lupus erythematosus. Lupus 2014; 23: 1085-1091.

17 Petri M, Orbai AM, Alarcón GS, et al. Derivation and validation of the Systemic Lupus International Collaborating Clinics classification criteria for systemic lupus erythematosus. Arthritis Rheum 2012; 64: 2677-2686.

18 Idrees MM, Saleemi S, Azem MA, et al. Saudi guidelines on the diagnosis and treatment of pulmonary hypertension: 2014 updates. Ann Thorac Med 2014; 9: Suppl. 1, S1-S15.

19 Galiè N, Humbert M, Vachiery JL, et al. ESC/ERS Guidelines for the diagnosis and treatment of pulmonary hypertension: the Joint Task Force for the Diagnosis and Treatment of Pulmonary Hypertension of the European Society of Cardiology (ESC) and the European Respiratory Society (ERS). Eur Respir J 2015; 46: 903-975.

20 Chinese Rheumatism Data Center. [Consensus on diagnosis and treatment of systemic lupus erythematosus associated pulmonary arterial hypertension in Chinese Adults]. Chin J Intern Med 2015; 54: 129-135.

21 Gladman DD, Ibañez D, Urowitz MB. Systemic lupus erythematosus disease activity index 2000. J Rheumatol 2002; 29: 288-291.

22 Schultz LR, Peterson EL, Breslau N. Graphing survival curve estimates for time-dependent covariates. Int $J$ Methods Psychiatr Res 2002; 11: 68-74.

23 Contal C, O'Quigley J. An application of changepoint methods in studying the effect of age on survival in breast cancer. Comput Stat Data Anal 1999; 30: 253-270.

24 Hachulla E, Jais X, Cinquetti G, et al. Pulmonary arterial hypertension associated with systemic lupus erythematosus: results from the French Pulmonary Hypertension Registry. Chest 2018; 153: 143-151.

25 Lefèvre G, Dauchet L, Hachulla E, et al. Survival and prognostic factors in systemic sclerosis-associated pulmonary hypertension: a systematic review and meta-analysis. Arthritis Rheum 2013; 65: 2412-2423.

26 D'Alonzo GE, Barst RJ, Ayres SM, et al. Survival in patients with primary pulmonary hypertension. Results from a national prospective registry. Ann Intern Med 1991; 115: 343-349.

27 Swiston JR, Johnson SR, Granton JT. Factors that prognosticate mortality in idiopathic pulmonary arterial hypertension: a systematic review of the literature. Respir Med 2010; 104: 1588-1607.

28 van Vollenhoven RF, Mosca M, Bertsias G, et al. Treat-to-target in systemic lupus erythematosus: recommendations from an international task force. Ann Rheum Dis 2014; 73: 958-967. 
29 Doria A, Gatto M, Zen M, et al. Optimizing outcome in SLE: treating-to-target and definition of treatment goals. Autoimmun Rev 2014; 13: 770-777.

30 Kylhammar D, Kjellström B, Hjalmarsson C, et al. A comprehensive risk stratification at early follow-up determines prognosis in pulmonary arterial hypertension. Eur Heart J 2018; 39: 4175-4181.

31 Boucly A, Weatherald J, Savale L, et al. Risk assessment, prognosis and guideline implementation in pulmonary arterial hypertension. Eur Respir J 2017; 50: 1700889.

32 Hoeper MM, Kramer T, Pan Z, et al. Mortality in pulmonary arterial hypertension: prediction by the 2015 European pulmonary hypertension guidelines risk stratification model. Eur Respir J 2017; 50: 1700740.

33 Zhao J, Bai W, Zhu P, et al. Chinese SLE Treatment and Research group (CSTAR) registry VII: prevalence and clinical significance of serositis in Chinese patients with systemic lupus erythematosus. Lupus 2016; 25: 652-657.

34 Huang C, Zhang S, Tian Z, et al. Could pulmonary arterial hypertension be an active index of systemic lupus erythematosus? A successful case of SLE-PAH cured by methylprednisolone pulse therapy. Lupus 2014; 23: 1533-1536.

35 Yasuoka H, Shirai Y, Tamura Y, et al. Predictors of favorable responses to immunosuppressive treatment in pulmonary arterial hypertension associated with connective tissue disease. Circ J 2017; 82: 546-554.

36 Humbert M, Sitbon O, Yaïci A, et al. Survival in incident and prevalent cohorts of patients with pulmonary arterial hypertension. Eur Respir J 2010; 36: 549-555. 